\title{
A Rare Presentation of Hard Palate Actinomycosis Mimicking a Neoplasm: Case Report
}

\author{
Ahmedbrahim A*, Medelhafed R, Hammouda Y, Oukessou Y, Sami R, \\ Reda A, Mohamed R and Mohamed M \\ Department of Otorhinolaryngology, King Hassan II University Hospital, Morocco
}

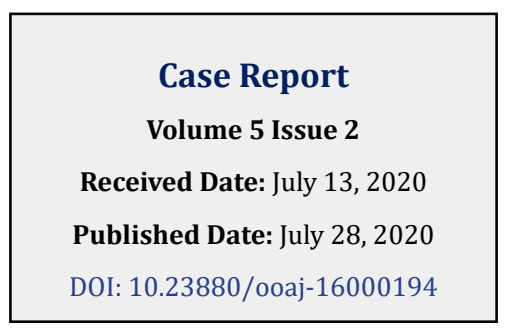

*Corresponding author: Ahmed Brahim Ahmedou, Department of Otorhinolaryngology, Head and Neck Surgery, King Hassan II University Hospital, Casablanca, Morocco, Tel: 00212635829293; Email: a.ahmedbrahim@gmail.com

\section{Abstract}

Actinomycosis is a rare, chronic, and slowly progressive granulomatous disease caused by Gram positive anaerobic bacteria. Actinomycosis of the head and neck is an uncommon and frequently misdiagnosed, actinomycosis can mimic many other diseases, including neoplasms, and the diagnosis may be difficult to establish. The following study reports a 70-year-old man with a painful ulcerations of the oral cavity for 6 months, he had been a smoker for 18 years; In addition, the patient had been diagnosed with epidermoid carcinoma of the lung 1 years previously, which was in remission after a radio chemotherapy. The diagnosis was confirmed by histopathological examination so he was treated with a regimen of oral ampicillin. Histopathological examination is the most reliable diagnostic tool to define the diagnosis. A high diagnostic index of suspicion for actinomycosis when ulcérative lesion of palate occurs benefits to the patient and the practitioner.

Keywords: Actinomycosis; Neoplasm; Oral cavity; Hard palate; Epidermoide carcinoma

\section{Introduction}

Actinomycosis is an uncommon and frequently misdiagnosed infection of the cervicofacial region. Most commonly, it presents as a slowly progressive, indolent, indurated infiltration with multiple abscesses, fistulas. A less common form is acute and rapidly progressive, with fever and a fluctuating swelling that resembles a typical pyogenic infection [1].

Actinomycosis can mimic many other diseases, including neoplasms, and the diagnosis may be difficult to establish [2]. Cervicofacial actinomycosis is the most common manifestation of the disease [3]. However palatal involvement is extremely rare here, the following reports a rare case of actinomycosis involving the hard palate mimicking a neoplasm.

\section{Case Report}

A 70-year-old man presented with a painful ulcerations of the oral cavity for 6 months duration. His medical history noted that he had been a smoker for 18 years, consuming 1 pack of cigarettes per day. In addition, the patient had been diagnosed with epidermoid carcinoma of the lung 1 year previously, which was in remission after a radio chemotherapy treatment. The patient had been referred to our department of Otorhinolaryngology and cervico facial surgery, Ibno Rochd universitary hospital of Casablanca, morocco; for palate ulcerative lesion. The oral examination (Figure 1), revealed an extensive ulcerative lesion on the hard palate that measured approximately $4 \mathrm{~cm}$ in diameter with yellow pseudomembrane deposit; we also noted a posterior erythema on the soft palate. The rest of ENT examination was normal and there was no evidence of cervical Lymphadenopathy. 


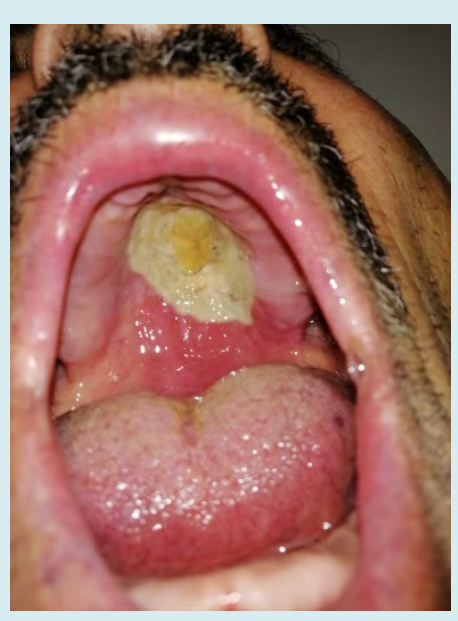

Figure 1: Ulcerative lesion of the hard palate with erythematous soft palate mucosa.

Laboratory investigations, including complete blood count, blood biochemistry, were within normal values. The CT scan (Figure 2) showed, an erasing of the palate mucosa relief with discontinue palate bone, and simultaneous antrochoanal polyp in the right nasal cavity. A biopsy of the lesion was scheduled under local anesthesia, and the result demonstrated squamous-lined mucosal tissue underlined by chronically inflamed granulation and fibrous tissue revealing many actinomycete colonies. The patient was treated by long term oral penicillin. During 6 month of the follow up we noted a resolving of symptoms and cicatrisation of the ulceration.

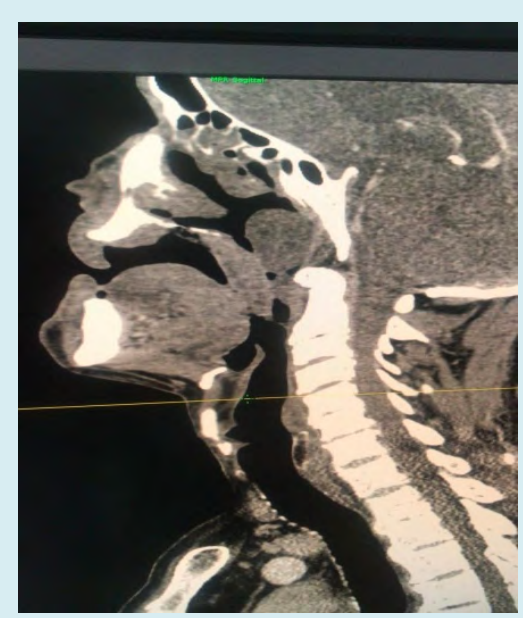

Figure 2: Sagital CT scan showed an ulceration of hard palate mucosa.

\section{Discussion}

Originally considered to be a fungus (mykos) because of its slow growth and filamentous appearance, it is currently generally accepted that Actinomyces are bacteria and are taxonomically classified accordingly as members of the order Actinomycetales and the family Actinomycetacae [4].

Actinomycosis is a rare, chronic, and slowly progressive granulomatous disease caused by filamentous Gram positive anaerobic bacteria from the Actinomycetaceae family. The bacteria can infect the skin and mucosal sites, such as the face, odontogenic and cervicofacial regions, respiratory tract, genitourinary tract, and digestive tract [5]. It is often misdiagnosed because it can mimic other conditions such as malignancy and tuberculosis [6]. The bacteria can infect the skin and mucosal sites, such as the face, odontogenic and cervicofacial regions, respiratory tract, genitourinary tract, and digestive tract [5]. It may occur at any age, however, essentially between age 30 and 60 . More frequent in men than women 4:1 [7]. Samuels and Martin separate 3 distinct presentations of Actinomycosis: acute painful swellings with duration shorter than 1 month; chronic longstanding infections with duration longer than 3 months; and unsuspected microbiologically proved actinomycotic lesions [8]. In our case, the medical history of his lung carcinoma and the clinical presentation as chronic ulcerative lesion of the palate; pushed us more to the hypothesis of metastatic lung carcinoma, so we immediately performed a biopsy of the lesion. A primary actinomycotic bone infection is uncommon but may occur in up to $12 \%$ of cases, because osseous invasion and destruction may result from spread of the infection from adjacent soft tissues, extraction sites, or fractures sites [4]. The CT scan result of paranasal sinuses described an ulcerative palate mucosa lesion who should be completed by histophatological examination to establish the diagnosis.

It had been demonstrated that histopathological examination is the most reliable diagnostic tool to define the diagnosis, so eliminate differentials diagnosis [9]. In this case the result of the histopathological examination was consistent with actinomycosis. Usually the treatment should be continued at least 6 months. Generally, oral actinomycosis infection and low bulk oral disease can be treated with oral agents for a shorter period [10].

In our case the lesion was not voluminous so we opted for unique oral treatment, moreover the results in the follow up was good. A high diagnostic index of suspicion for actinomycosis when ulcérative lesion of palate with occurs benefits the patient and the practitioner. A prolonged course of penicillin is the gold standard treatment.

\section{Conclusion}

Actinomycosis is a relatively rare infection caused by saprophytic bacteria of the oral cavity. This infection remains 


\section{Otolaryngology Open Access Journal}

largely unknown to most clinicians because of its different presentations, and palatal involvement is extremely rare. However, health care professionals should be aware of the presence of ulcerative, destructive oral lesions because they can mimic malignant conditions.

\section{References}

1. De D, Dogra S, Kanwar AJ, Saikia UN (2011) Actinomycosis presenting as a destructive ulcerated plaque on the palate and gingiva. J Am Acad Dermatol 65(6): 12351236.

2. Bennoff DF (1984) Actinomycosis: Diagnostic and therapeutic considerations and a review of 32 cases. Laryngoscope 94(9): 1198-1217.

3. Nielsen PM, Novak A (1987) Acute cervico-facial actinomycosis. Int J Oral Maxillofac Surg 16(4): 440-444.

4. Goldberg MH (2003) Diagnosis and treatment of cervicofacial actinomycosis. Oral Maxillofac Surg Clin N Am 15(1): 51-58.
5. Elzein F, Kharraz R, Arab N, Alotaibi F, Almohaya A, et al. (2019) A case series of actinomycosis from a single tertiary care center in Saudi Arabia. IDCases 15: e00521.

6. Wong VK, Turmezei TD (2011) Actinomyces. British Med $\mathrm{J}$.

7. Foster SV, Demmier GJ, Hawkins EP (1993) Pediatric cervico-facial actinomycosis. South Med J 86: 1147-1150.

8. Samuels RH, Martin MV (1988) A clinical and microbiological study of Actinomycetes in oral and cervicofacial lesions. Br J Oral Maxillofac Surg 26(6): 458-463.

9. Sakallioglu U, Acikgoz G, Kirtiloglu T, Karagoz F (2003) Rare lesions of the oral cavity: case report of an actinomycotic lesion limited to the gingiva. J Oral Sci 45(1): 39-42.

10. Martin MV (1984) The use of oral amoxicillin for the treatment of actinomycosis A clinical and in vitro study. Br Dent J 156(7): 252-254. 